\title{
Maverick dark matter at colliders
}

\author{
Maria Beltrán \\ Department of Astronomy and Astrophysics and Enrico Fermi Institute, \\ University of Chicago, 5640 S. Ellis Ave., Chicago, Illinois 60637
}

Dan Hooper

Theoretical Astrophysics, Fermi National Accelerator Laboratory, Box 500, Batavia, Illinois 60637 and Department of Astronomy and Astrophysics and Kavli Institute for Cosmological Physics, University of Chicago, 5640 S. Ellis Ave., Chicago, Illinois 60637

\author{
Edward W. Kolb \\ Department of Astronomy and Astrophysics, Enrico Fermi Institute, \\ and Kavli Institute for Cosmological Physics, University of Chicago, 5640 S. Ellis Ave., Chicago, Illinois 60637 \\ Zosia A. C. Krusberg \\ Department of Physics and Enrico Fermi Institute, \\ University of Chicago, 5640 S. Ellis Ave., Chicago, Illinois 60637
}

Tim M. P. Tait

Department of Physics and Astronomy, University of California, Irvine, California 92697

\begin{abstract}
Assuming that dark matter is a weakly interacting massive particle (WIMP) species $X$ produced in the early Universe as a cold thermal relic, we study the collider signal of $p p$ or $p \bar{p} \rightarrow \bar{X} X+$ jets and its distinguishability from standard-model background processes associated with jets and missing energy. We assume that the WIMP is the sole particle related to dark matter within reach of the LHC - a "maverick" particle - and that it couples to quarks through a higher dimensional contact interaction. We simulate the WIMP final-state signal $X \bar{X}+$ jets and dominant standard-model (SM) background processes and find that the dark-matter production process results in higher energies for the colored final state partons than do the standard-model background processes. As a consequence, the detectable signature of maverick dark matter is an excess over standard-model expectations of events consisting of large missing transverse energy, together with large leading jet transverse momentum and scalar sum of the transverse momenta of the jets. Existing Tevatron data and forthcoming LHC data can constrain (or discover!) maverick dark matter.
\end{abstract}

PACS numbers: $13.85 . \mathrm{Rm}, 14.80 .-\mathrm{j}, 98.80 . \mathrm{Cq}, 95.35 .+\mathrm{d}$

\section{INTRODUCTION}

Compelling observational evidence for the existence of dark matter has been found on a wide range of astronomical scales. However, only a few things are presently known about the physical properties of dark matter: its relic density has been determined very precisely by the WMAP experiment to be $\Omega_{X} h^{2}=0.1109 \pm 0.0056$ [1] , and observations suggest that it is non-baryonic, cold, dissipationless, and stable on time scales on the order of the age of the universe. Many dark-matter candidates have been proposed. Among these, weakly interacting massive particles (WIMPs) are particularly compelling: they are predicted by many theories of beyond the standard-model (BSM) physics and can naturally produce the observed relic dark-matter density through thermal processes.

Many previous studies have sought to constrain the properties of various WIMP candidates using collider data. Most of these have studied WIMP candidates within specific theoretical frameworks such as supersymmetry [2] or models with warped [3] or universal [4] extra dimensions. The constraints have normally been imposed by various collider bounds, such as the bounds on the Higgs mass, the invisible $Z$ width, the masses of new charged and colored particles, the masses of new charged and neutral gauge bosons, flavor-changing neutral currents, the branching ratios of $b \rightarrow s \gamma$ and $B_{s} \rightarrow \mu^{+} \mu^{-}$, the anomalous magnetic moment of the muon, and other electroweak precision measurements [5]. Stringent constraints on various particle models have been obtained in such analyses (see e.g., Ref. [6] for an analysis of mSUGRA parameters and Ref. 7] for an analysis of large extra dimensions parameters).

The theoretical frameworks to which these WIMP candidates belong are often both theoretically well motivated and compelling. However, given that all of these theories still lack experimental support, we cannot exclude the possibility that dark matter belongs to some other, yet unidentified, theory. Additionally, given that the first observations of dark matter may come from direct- or indirect-detection experiments, which may only provide information about the general properties of the dark-matter particle without offering a way to distinguish between underlying theories, 
it is important to remain unbiased about the nature of dark matter. For this reason, model-independent studies of dark-matter phenomenology using effective field theory can be particularly important.

In fact, examining the primary experimental analyses of our favorite theories reveals a common feature of such constraints: they are relatively insensitive to the nature of the dark-matter particles per se, and instead probe the properties of the new, exotic colored and/or charged states that accompany the dark-matter particle. Such states are more amenable to experimental probes because they have large standard-model gauge interactions and are thus more easily produced. However, if our primary motivation is to learn about the nature of dark matter, they are something of a distraction. Further, when such charged states are somewhat heavier than the dark matter itself, the only signals accessible to colliders (at least in early data sets) may be the production of new, heavy SM-charged particles.

In a recent paper [8], an effective field theory approach was used to evaluate the constraints on generic WIMP candidates from direct-detection experiments and the prospects for a signal in future indirect-detection experiments. Several WIMP candidates with specified spin and interaction forms with standard-model fermions were found to be excluded on the basis of direct-detection experiments. An interesting possibility - a fermionic WIMP with an axialvector coupling to standard-model fermions - was not ruled out by direct-detection experiments, and products of its annihilation in the Sun are expected to be eventually detectable by a neutrino detector such as IceCube. Motivated by this result, we study dark matter at accelerators by investigating the production and detection prospects of this WIMP candidate at the Tevatron and at the LHC.

Our effective field theory description of dark matter is largely model independent, but differs in important ways from previous model-independent analyses [9, 10]. These previous works take the annihilation cross section as inferred from the relic density, time-reverse it into a collider production process (taking into account subtleties related to spin and flux factors appropriately), and use factorization theorems to produce SM radiation. Our effective field theory description pays the price of being more model-dependent, but has the advantage that it can describe relativistic production, which may differ substantially from the nonrelativistic cross section relevant to describe freeze out. This difference is particularly important if the nonrelativistic cross section appropriate for the freeze-out calculation is $p$-wave suppressed, as in the case of the axial-vector interactions we will study. In fact, this difference will contribute to our somewhat more optimistic conclusions concerning the potential for observing WIMPs at the LHC.

\section{EFFECTIVE FIELD THEORY}

We assume that our WIMP candidate is the only new particle species within reach of the LHC (a "maverick" particle); this allows us to describe its interaction with standard-model quarks accurately in terms of an effective field theory whose degrees of freedom consist of the standard-model (SM) particles plus the WIMP itself. For the purposes of the discussion, we also specialize to the case of a Dirac fermion. The effective field theory consists of the SM Lagrangian plus kinetic terms for the dark matter $X$ and a set of effective four-Fermion interactions between $X$ and the quarks $q=u, d, s, c, b, t$,

$$
\mathcal{L}=\mathcal{L}_{S M}+i \bar{X} \gamma^{\mu} \partial_{\mu} X-M_{X} \bar{X} X+\sum_{q} \sum_{i, j} \frac{G_{q i j}}{\sqrt{2}}\left[\bar{X} \Gamma_{i}^{X} X\right]\left[\bar{q} \Gamma_{q}^{j} q\right]
$$

where the sums $i, j$ are over scalar, pseudoscalar, vector, axial vector, and tensor interactions (in Lorentz-invariant combinations) described by the operators $\{\Gamma\}$. We will assume that the interaction is dominated by only one of the above forms. ${ }^{1}$ The mass dimension of $G_{q i j}$ is minus two. Although it would be straightforward to account for WIMPs coupling to leptons, for simplicity, we do not take this possibility into account in our analysis, and we further specialize to the case in which the WIMP couplings are independent of the quark flavor, $G_{q i j}=G_{i j}$. With these assumptions, and the assumption that the dark-matter density is determined by the calculation of the thermal relic abundance of the $X \mathrm{~s}$, the various $G_{i}$ 's are determined to high precision. Even if the WIMP proves not to be a thermal relic, the effective field theory (with the $G$ 's uncorrelated from $M_{X}$ ) may still provide a useful language to describe WIMP coupling to SM particles.

In the case where the WIMP interaction is spin independent, the allowed range for the WIMP mass and coupling constant is very tightly constrained from direct-detection search experiments [8]. However if the $X q \rightarrow X q$ scattering is spin dependent, then the corresponding direct-detection limits are too weak to exclude any of the acceptable

\footnotetext{
${ }^{1}$ As in Ref. [8], we do not consider WIMP couplings to Higgs or gauge bosons. More general actions including such terms can be found in Ref. [12].
} 


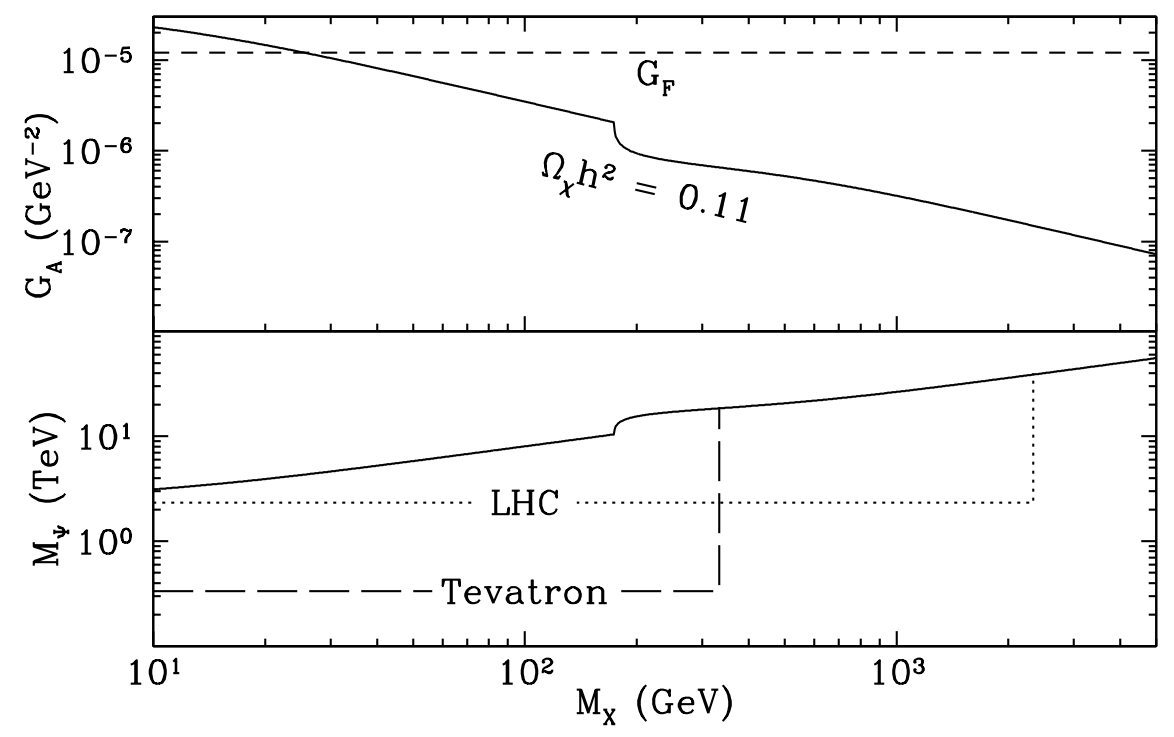

FIG. 1: The upper panel indicates the value of $G_{A}$ as a function of the WIMP mass $M_{X}$ necessary to result in a relic abundance of $\Omega_{X} h^{2}=0.11$ if the interaction Lagrangian is given by Eq. (2). Shown for comparison is the value of the Fermi coupling constant, $G_{F}$. For the effective field theory of Sect. II to be valid, the mass of $\Psi_{\mu}$ in Eq. (3) must be below the solid curve and above the dashed (Tevatron) and dotted (LHC) horizontal lines in the bottom panel. The vertical dashed and dotted lines indicate the rough kinematic reach for dark-matter production for the indicated hadron collider. The kinks in the curves around $M_{X}=170 \mathrm{GeV}$ correspond to the opening of the top quark production channel, allowing for weaker couplings (top panel) and consequently requiring larger $M_{\psi}^{\prime} s$ (bottom panel).

WIMP mass and coupling constant combinations. Therefore, we will consider interactions that yield a spin-dependent scattering cross section. In particular, we simplify the consideration to the axial-vector case and consider a Lagrangian

$$
\mathcal{L}_{i n t}=\frac{G_{A}}{\sqrt{2}} \sum_{q}\left[\bar{X} \gamma^{\mu} \gamma^{5} X\right]\left[\bar{q} \gamma_{\mu} \gamma_{5} q\right]
$$

The coupling constant $G_{A}$ is obtained for a WIMP of a given mass from the requirement that it leads to the correct relic density found by WMAP within the standard thermal freezeout framework (see, e.g., Ref. [13]). The resulting $G_{A}$ as a function of $M_{X}$ is shown in the upper panel of Fig. 1. Our primary assumption that the WIMP is the only new particle within LHC reach also ensures that there are no resonances or coannihilations to complicate the standard analysis of the relic density.

While we work with an effective low-energy field theory, it is instructive to discuss possible ultraviolet (UV) completions of our theory. One possible completion would involve a massive intermediate vector boson $\Psi_{\mu}$ with mass much larger than any other mass or energy scale associated with the low-energy effective field theory. Then $G_{A}$ of the effective field theory is related to parameters of the UV complete theory by

$$
\frac{G_{A}}{\sqrt{2}}=\frac{g_{q} g_{X}}{M_{\Psi}^{2}},
$$

where $g_{q}\left(g_{X}\right)$ is the coupling constant for the $\Psi-\bar{q}-q(\Psi-\bar{X}-X)$ interaction. With the requirement that the dimensionless coupling constants $g_{q}$ and $g_{X}$ are smaller than $4 \pi$, for a given value of $G_{A}\left(M_{X}\right)$ (set by the relic density) there is a maximum value of $M_{\Psi}$ such that the UV completion admits a perturbative description. This is shown by the solid curve in the bottom panel of Fig. 1. For the effective theory to make sense, $\Psi_{\mu}$ must be massive enough so as to not be directly accessible at the energies of interest. At a hadron collider of center of mass energy $E$, this requirement ultimately depends on the details of the relevant parton distribution functions (PDFs) and the collected luminosity. A very rough estimate is furnished by $M_{\Psi} \gtrsim E / 3$. The resulting lower limits for the Tevatron and the LHC are shown by the horizontal lines in the bottom panel of Fig. 1. Also shown as the vertical lines in the bottom panel of Fig. 1 is the approximate kinematic mass reach for $X \bar{X}$ production at the Tevatron and LHC. We will discuss detailed estimates for the discovery potential of $X$ as a function of its mass in the sections below. 

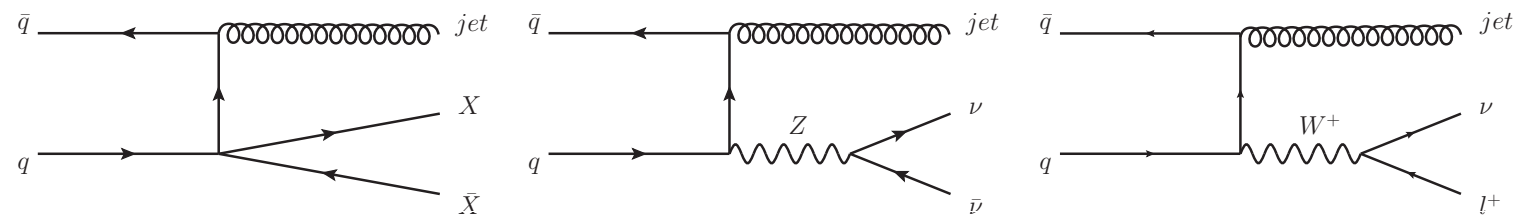

FIG. 2: Representative Feynman diagrams (at the parton level) for the processes $p p \rightarrow X \bar{X}+$ jet (left), $p p \rightarrow \nu \bar{\nu}+$ jet (center), and $p p \rightarrow l^{+} \nu+$ jet (right).

\section{COLLIDER SIGNAL AND BACKGROUND}

\section{A. Processes}

Our effective theory for WIMP - SM interactions leads to the production of WIMPs at colliders through the process

$$
p p(p \bar{p}) \rightarrow X \bar{X}
$$

However, this process is worthless as a discovery mode at a hadron collider because it contains no visible trace that a hard scattering took place at all. Consequently, we turn to the process in which WIMPs are produced together with a hard parton, ${ }^{2}$

$$
p p(p \bar{p}) \rightarrow X \bar{X}+\text { jets. }
$$

While this process is formally higher order in perturbation theory, the hard jet(s) of hadrons provides a trigger that a hard scattering actually took place, with the WIMPs "seen" as missing momentum against which the jet recoils.

The dominant SM physics backgrounds consist of electroweak processes, such as $Z+$ jets, where the $Z$ decays into a pair of neutrinos,

$$
p p(p \bar{p}) \rightarrow \nu \bar{\nu}+\text { jets }
$$

as well as $W^{ \pm}+$jets where the $W$ decays into a neutrino and a charged lepton,

$$
p p(p \bar{p}) \rightarrow l^{-} \bar{\nu}+\text { jets and } p p(p \bar{p}) \rightarrow l^{+} \nu+\text { jets, }
$$

and the charged lepton either falls outside of the acceptance range of the detector or is lost inside a jet. At the LHC, we also consider the background from $t \bar{t}$ production:

$$
p p \rightarrow t \bar{t} \rightarrow W^{+} b W^{-} \bar{b}
$$

whose decays again produce $W$ bosons. There are additional "QCD" backgrounds that arise from purely stronginteraction processes in which mismeasurement leads to fake missing transverse momentum. This background depends intricately on the details of the detector, and is beyond the scope of our ability to model properly. That said, we will apply stiff missing momentum cuts and require the leading jet to be acollinear with the missing transverse momentum. Both cuts should help minimize the sensitivity to detector details on our search proposal.

Representative parton-level Feynman diagrams for signal and electroweak background processes are shown in Fig. 2. We simulate the signal and background events using the MadEvent package [14], in which we have implemented a Dirac fermion WIMP which interacts with the SM through the maverick interaction of Eq. (2). After MadEvent generates the hard scattering process, Pythia [15] is called to simulate parton showering and hadronization, and PGS (with the generic Tevatron and LHC detector models) provides an estimate of the detector effects [16].

While the dominant corrections to the kinematics from higher orders of perturbation theory are captured by the parton shower, higher order contributions also correct the over-all rates of the processes. We improve our estimates by applying a flat $K$-factor to the SM background rates,

$$
K \text {-factor }=\frac{\sigma_{\mathrm{NLO}}}{\sigma_{\mathrm{LO}}} .
$$

\footnotetext{
${ }^{2}$ Recently, Ref. [11] studied WIMPs produced at the LHC together with a hard photon.
} 
A flat $K$-factor is known to work reasonably well for both $W+$ jets and $Z+$ jets, and their values are similar for both processes [17],

$$
\begin{array}{r}
\text { Tevatron: } K_{W, Z}=1.20 \\
\text { LHC: } K_{W, Z}=1.32 .
\end{array}
$$

While we expect that a similar $K$-factor applies to the WIMP signal process, in the absence of an explicit computation and to be conservative, we use leading order rates for the signal. The $K$-factor for $t \bar{t}$ at the LHC is taken to be [18]

$$
K_{t \bar{t}}=1.54
$$

\begin{tabular}{lcccc}
\hline \hline Collider & Process & $M_{\chi}(\mathrm{GeV})$ & Cross section before cuts $(\mathrm{pb})$ & Cross section after cuts $(\mathrm{pb})$ \\
\hline \hline Tevatron & $p \bar{p} \rightarrow X \bar{X} J$ & 5 & $4.18 \times 10^{1}$ & $2.43 \times 10^{-2}$ \\
& 10 & $1.74 \times 10^{1}$ & $1.05 \times 10^{-2}$ \\
& 25 & $4.16 \times 10^{0}$ & $2.25 \times 10^{-3}$ \\
& & 50 & $9.62 \times 10^{-1}$ & $9.62 \times 10^{-4}$ \\
& & - & $1.64 \times 10^{2}$ & $1.15 \times 10^{-2}$ \\
& $p \bar{p} \rightarrow \nu \bar{\nu} J$ & - & $4.19 \times 10^{2}$ & $4.20 \times 10^{-3}$ \\
& $p \bar{p} \rightarrow l^{-} \bar{\nu} J$ & - & $4.20 \times 10^{2}$ & $8.43 \times 10^{-3} a$ \\
\hline LHC & $p \bar{p} \rightarrow l^{+} \nu J$ & 10 & $9.42 \times 10^{2}$ & $1.84 \times 10^{1}$ \\
& $p p \rightarrow X \bar{X} J$ & 50 & $6.47 \times 10^{1}$ & $1.27 \times 10^{0}$ \\
& & 100 & $1.51 \times 10^{1}$ & $3.30 \times 10^{-1}$ \\
& & 500 & $1.18 \times 10^{-1}$ & $3.82 \times 10^{-3}$ \\
& $p p \rightarrow \nu \bar{\nu} J$ & - & $2.52 \times 10^{3}$ & $4.16 \times 10^{-1}$ \\
& $p p \rightarrow l^{-} \bar{\nu} J$ & - & $4.00 \times 10^{3}$ & $7.05 \times 10^{-2}$ \\
& $p p \rightarrow t \bar{t}$ & - & $5.26 \times 10^{3}$ & $1.68 \times 10^{-1}$ \\
\hline \hline
\end{tabular}

${ }^{a}$ Because $W$ interactions are left-handed (and the light fermions are effectively massless), there are different spin correlations in the $p_{T}$ distributions for $l^{+}$and $l^{-}$. Charged leptons produced by $W^{+}$'s are anti-particles, with right-handed helicity, while charged leptons produced by $W^{-}$'s are particles, with left-handed helicity. The difference in the $p_{T}$ distributions results in different efficiencies to pass our cuts that veto high- $p_{T}$ leptons. (This also applies to the cross sections for the analogous processes at the LHC.)

TABLE I: Cross sections for the production of $X \bar{X}+$ jets for the indicated WIMP masses and the background processes specified in Eqs. (6) - (7) at the Tevatron (center-of-mass energy $\sqrt{s}=1.96 \mathrm{TeV}$ ) and the LHC (center-of-mass energy $\sqrt{s}=14 \mathrm{TeV}$ ), before and after cuts.

Inclusive cross sections for signal and background processes at the Tevatron (a $p \bar{p}$ collider with $\sqrt{s}=1.96 \mathrm{TeV}$ ) and the LHC (a $p p$ collider with $\sqrt{s}=14 \mathrm{TeV}$ ) are given in Table \. Minimal cuts of $p_{T} \geq 20 \mathrm{GeV}$ and $|\eta| \leq 3.6$ (Tevatron) $|\eta| \leq 2.5$ (LHC) are imposed on the hard parton, in order to render the rates IR-safe. The quantity $\eta$ is the pseudo-rapidity of the jet. Such loose cuts are marginally realistic at the Tevatron and completely unrealistic at the LHC. We will discuss our actual (realistic) analysis cuts below.

As the $X$ mass increases, the signal from $X \bar{X}+$ jet decreases. This is in part because we adjust $G_{A}$ together with $M_{X}$ to hold the thermal relic density fixed. The relic density is inversely proportional to the cross section for $X \bar{X} \rightarrow q \bar{q}$, which scales roughly as $\sigma \propto G_{A}^{2} M_{X}^{2}$, so keeping the relic density fixed implies $G_{A}\left(M_{X}\right) \propto M_{X}^{-1}$. In addition, higher energy partons are required to produce heavier $X$ particles, and the parton distribution functions fall steadily with the energy of the parton. The separation of the two effects is illustrated in Fig. 3, along with the cross sections as a function of $M_{X}$.

\section{B. Analysis and Cuts}

Our missing energy signature typically contains only jets as visible particles in the detector. In order to trigger on the events, we require that the leading jet is central and has a minimum transverse momentum. These cuts are $p_{T, \text { jet }}>250 \mathrm{GeV},|\eta| \leq 3.6$ at the Tevatron or $p_{T, \text { jet }}>450 \mathrm{GeV},|\eta| \leq 2.5$ at the LHC. The $p_{T}$ cuts are somewhat greater than those sufficient for an inclusive jet trigger at CDF and D $\emptyset$ at the Tevatron, or ATLAS and CMS at the LHC. In order to select events in which WIMPs may have been produced, we require a missing energy cut of $\mathbb{E}_{T}>30$ 


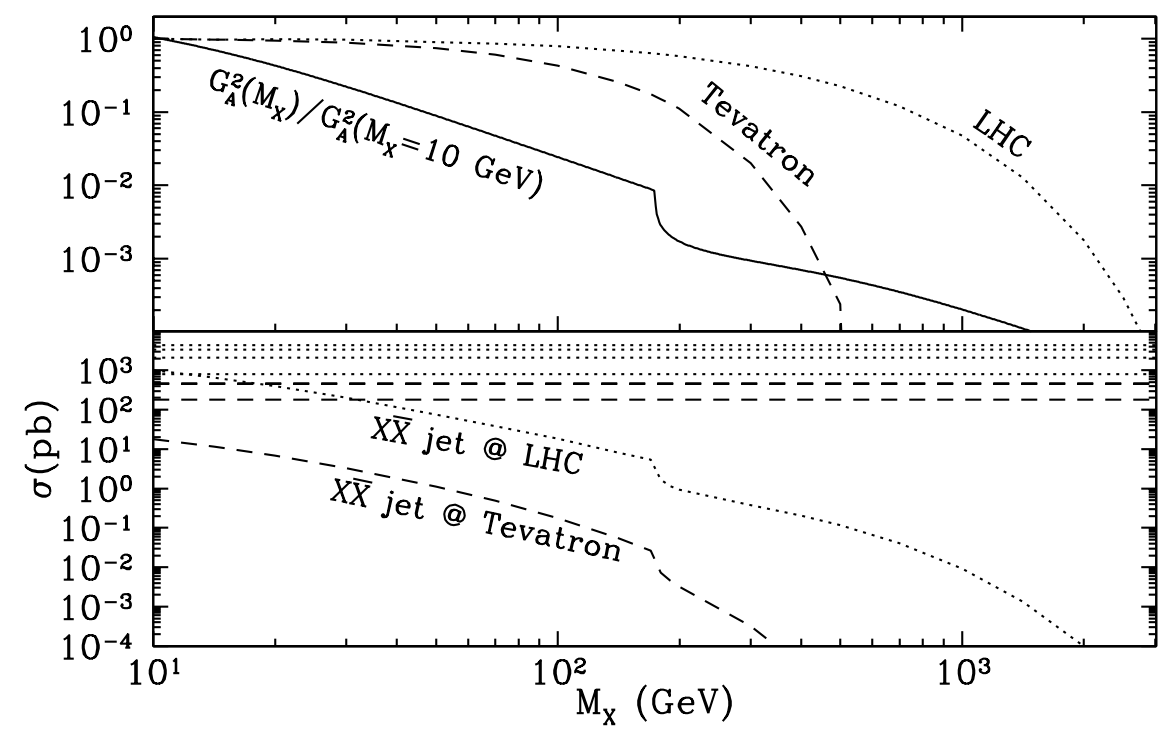

FIG. 3: Upper panel: Suppression factors as a function of the WIMP mass. The broken curves track the kinematic and parton flux suppression for the indicated hadron colliders, normalized to unity at a mass of $10 \mathrm{GeV}$. The solid curve represents the suppression due to the decrease in $G_{A}$ as $M_{X}$ increases (again normalized to unity at a mass of $10 \mathrm{GeV}$ ). The total suppression as a function of mass is the product of the two factors. Lower panel: $X \bar{X}+$ jet signal cross section as a function of the $X$ mass and the cross sections for the background processes at the LHC (dottel lines) and the Tevatron (dashed lines).The background processes for the indicated colliders are, from top to bottom, $l^{+}$jet, $l^{-}$jet, $\nu \bar{\nu}$ jet, and $t \bar{t}$. (The cross sections for $l^{+}$jet and $l^{-}$ jet at the Tevatron are nearly indistinguishable.) The kinks in the curves around $M_{X}=170 \mathrm{GeV}$ correspond to the opening up of the top quark production channel, allowing for weaker couplings (top panel) and correspondingly lower cross sections (bottom panel).
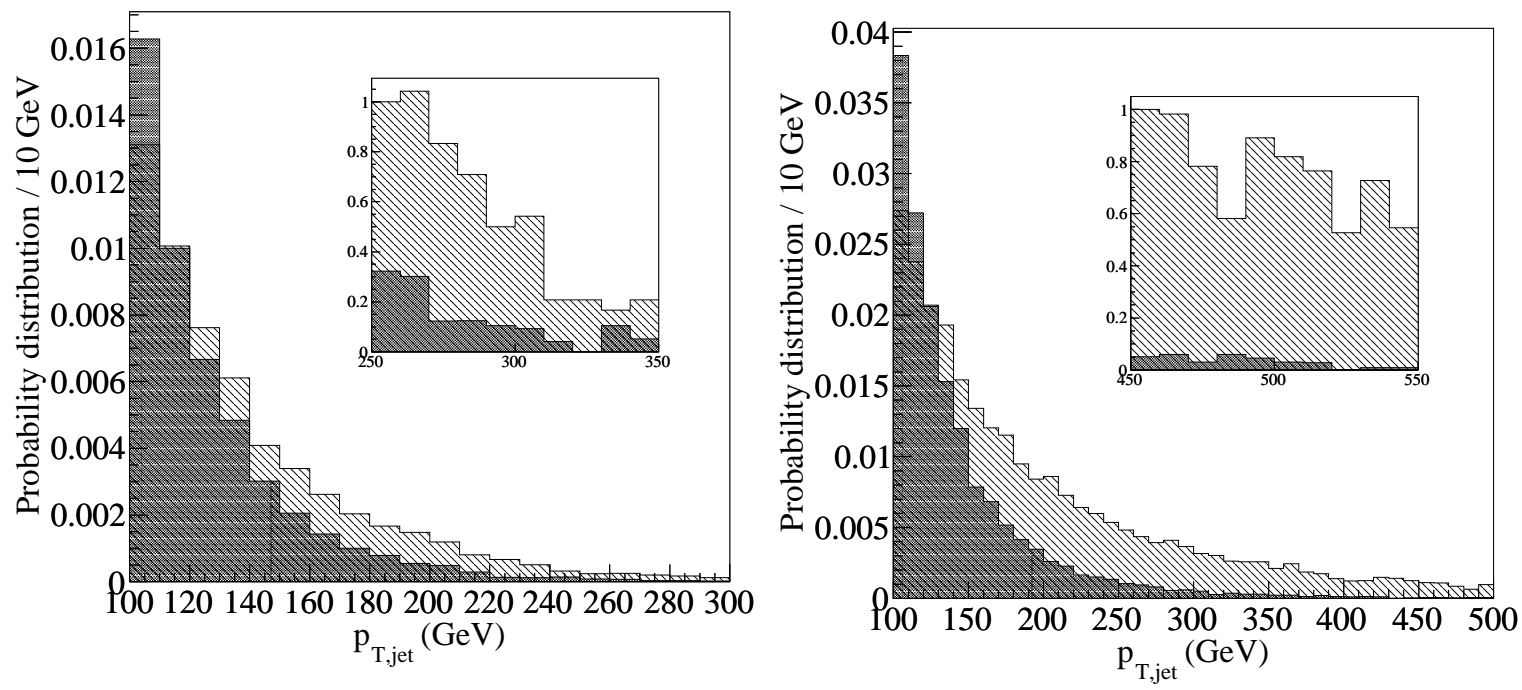

FIG. 4: The normalized distributions of the transverse momentum of the leading parton for the standard-model background (dark region) and the maverick dark-matter signal (light region) at the Tevtron (left) and the LHC (right). The insert in each the figure shows the distribution of events for which the leading parton has transverse momentum satisfying $p_{T} \geq p_{T, \text { min }}$. The signal has been generated with $M_{X}=5 \mathrm{GeV}$ at the Tevatron and $M_{X}=50 \mathrm{GeV}$ at the LHC. 
$\mathrm{GeV}$ (both colliders). To minimize the contribution of fake missing energy from jet mismeasurements, we require that the $\mathbb{E}_{T}$ and leading jet point in different azimuthal directions, $\left|\phi_{\text {jet }}-\phi_{\mathbb{E}_{T}}\right|>0.5 \mathrm{rad}[19]$.

In order to reduce backgrounds containing missing energy from processes where $W$ bosons are produced, we veto events containing one or more energetic isolated charged leptons (either $e^{ \pm}, \mu^{ \pm}$, or hadronically reconstructed $\tau^{ \pm}$). We remove such events for which a charged lepton has transverse momenta $p_{T, l^{ \pm}} \geq 10 \mathrm{GeV}$ and is further separated from all jets by $\Delta \mathcal{R}=\sqrt{(\Delta \eta)^{2}+(\Delta \phi)^{2}}>0.4$. Softer leptons may be inefficiently reconstructed by the detector, and those closer to jets may be "lost" inside them or result from heavy flavor decays inside the jet hadrons. Our implementation of the charged lepton cuts is very similar to those used by a recent CDF monojet search [20], and we are able to reproduce the post-cut event number quoted by CDF. In addition, to help with the $t \bar{t}$ background, we reject events in which one or more of the jets is tagged as containing a bottom quark.

Maverick dark matter can interact with SM particles only via non-renormalizable interaction terms. Such terms produce cross sections which fall less slowly compared to those mediated entirely by SM interactions. Thus, we expect the energy of the associated jet in $X \bar{X}+$ jet will typically be more than the energy associated with $W+$ jet or $Z+$ jet, providing a handle that potentially can be used to distinguish the signal from the background. In Fig. 4 we plot (at the parton level) the $p_{T}$ of the parton produced in the hard scattering in events from $X \bar{X}+$ jet and the SM backgrounds. The distributions are normalized to unity to emphasize the difference in the shapes with respect to the parton $p_{T}$.

Corrections from higher orders in perturbative QCD may modify the energy distribution of the hard parton. While the parton shower correctly captures higher order corrections to the kinematics resulting from soft or collinear radiation, it fails when the additional parton is radiated at wide angles with respect to its parent. Improved simulation of the kinematic distributions is possible by matching Monte Carlo samples with exact leading order matrix elements for additional radiation in the hard scattering process to those for lower order processes. These "jet matched samples" have the virtue of using the parton shower to resum soft and collinear logarithms while incorporating the exact matrix elements for perturbative radiation 21]. In missing energy observables, they tend to enhance tails in the missing energy distributions of both signal and background processes 22. While a detailed simulation of such effects is beyond the scope of this work, we expect it will shift both the signal and background $\mathbb{E}_{T}$ distributions by a similar factor, and should ultimately be included when analyzing collider data.

The precise manifestation of the harder partons in the signal events depends to some degree on the details of the analysis. Harder partons have more energy to radiate, and produce a spray of more energetic particles, some of which may form separate additional jets. We find that for the cone algorithm (with cone size $R=0.4$ ), the signal events typically end up with three or more jets after parton showering, and the the backgrounds peak at two (but both signal and background have substantial tails). The distribution of the number of jets of both signal and background processes is shown in Fig. 5. The higher energies of the primary partons in the signal process reflects itself in a higher jet multiplicity. This in turn reflects itself in larger typical values of the $H_{T}=\sum\left|\vec{p}_{T}\right|+\mathbb{E}_{T}$ variable.

In Fig. 6 we present the distributions of the $p_{T}$ of the leading jet, $H_{T}$, and $\mathbb{E}_{T}$ for signal and background at the Tevatron. For the signal, we have chosen a WIMP mass of $5 \mathrm{GeV}$. The coupling $G_{A}$ is fixed such that $X$ reproduces the correct thermal relic density, and the normalization corresponds to an integrated luminosity of $\int L \mathrm{dt}=10 \mathrm{fb}^{-1}$. The position of our primary cut $p_{T} \geq 250 \mathrm{GeV}$ is indicated by the dashed line, and clearly provides very effective separation of signal from background. Fig. 7 shows the same three distributions for the $\sqrt{s}=14 \mathrm{TeV}$ LHC, where the signal corresponds to a WIMP of mass $50 \mathrm{GeV}$, and the integrated luminosity is $\int L \mathrm{dt}=100 \mathrm{fb}^{-1}$.

\section{PROJECTED SENSITIVITY}

Ideally, one would compare the different shapes of the various observables: $p_{T}$ of the leading jet, $\mathbb{E}_{T}$, and $H_{T}$ and/or the number of jets between the collider data and the predictions for the SM backgrounds, and either put a limit on a combination of the mass of the maverick dark-matter particle and its interaction strength, or conclude that there is evidence for a signal of physics beyond the Standard Model. Such a shape-based analysis is beyond the scope of this work.

To estimate the approximate reach, we treat the signal as a counting experiment, and ask for which $M_{X}$ (with $G_{A}$ continuing to be set for each $M_{X}$ in order to hold the thermal relic density at its measured value) the signal would be large enough to represent a statistically significant deviation above the background. In practice, this is probably a reasonable approximation to the shape analysis to which we alluded above. Since we have seen that for high enough leading jet $p_{T}$ the background falls rapidly while the signal falls much more slowly, we expect that a rate analysis is much like a crude shape analysis with a single high $p_{T}$ bin.

The backgrounds may be controlled by using data themselves. The $Z+$ jets process can be studied through the visible $Z \rightarrow \ell^{+} \ell^{-}$decay mode, which is relatively easy to reconstruct thanks to the charged leptons, and allows one to measure the associated jet distributions and the $p_{T}$ of the $Z$ itself (which in the missing energy analysis is the 

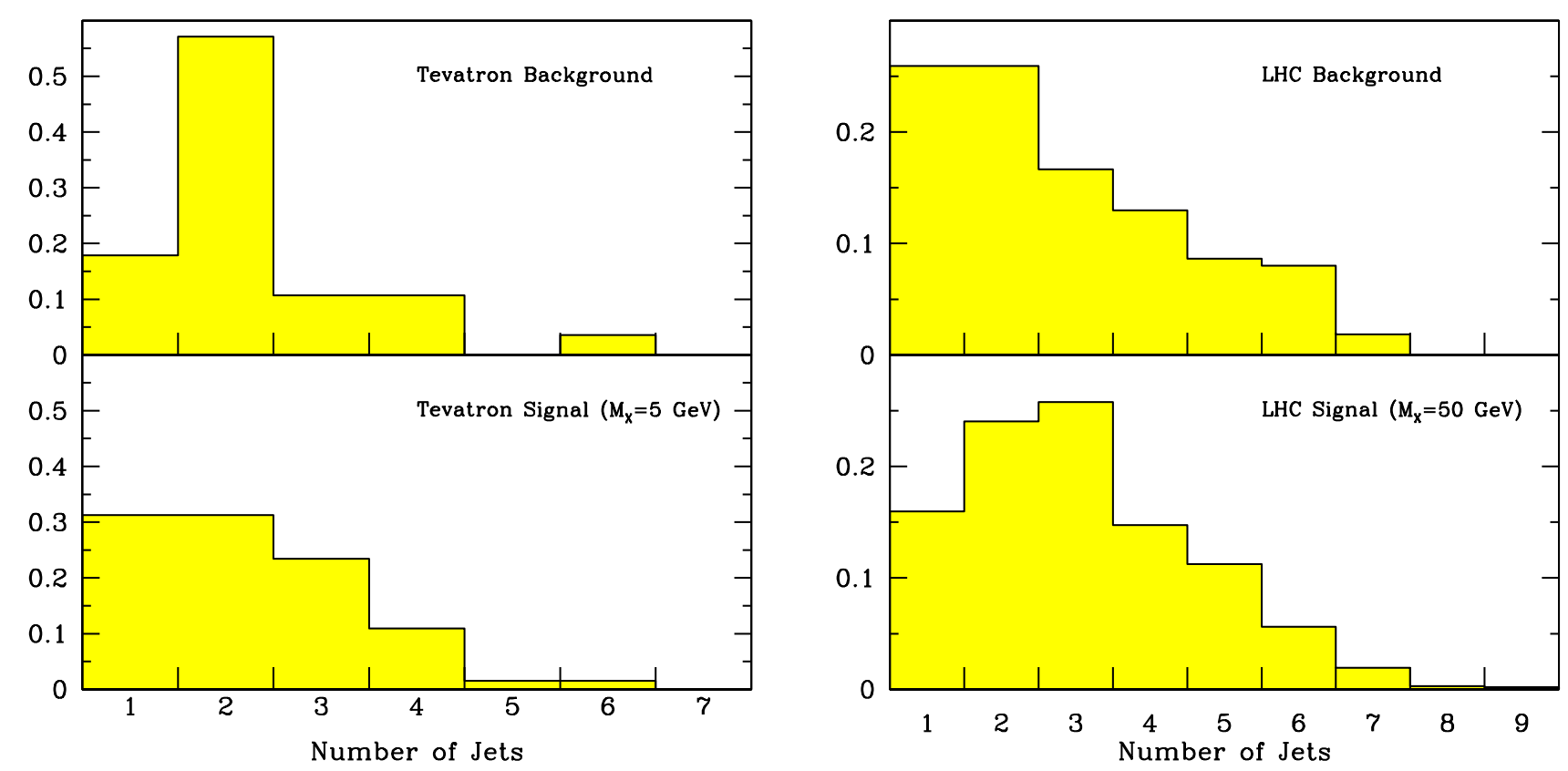

FIG. 5: Distributions for the number of jets (after the $p_{T}$ and $\mathbb{E}_{T}$ cuts discussed in the text) for signal and background processes at the Tevatron for a WIMP mass of $5 \mathrm{GeV}$ (left) and the LHC for a WIMP mass of $50 \mathrm{GeV}$ (right). For each case, the sum of the background processes are shown in the upper panels, and the signal distributions in the lower panels.

$\left.\mathbb{E}_{T}\right)$. Similarly, one can study the $W+$ jets process for cases in which the charged lepton can be reconstructed, and extrapolate into the regions where the charged lepton is missed.

From the signal and background rates (after cuts) in Table I, we determine the expected number of signal events $(S)$ and the sum of the background events $(B)$ expected with integrated luminosities of $10 \mathrm{fb}^{-1}$ (at the Tevatron) and $100 \mathrm{fb}^{-1}$ (at the $14 \mathrm{TeV}$ LHC), as a function of the WIMP mass. After cuts, the expected background rates are still expected to produce 100 events or more, and Gaussian statistics may be safely applied. Requiring a signal which is significant at the $5 \sigma, S / \sqrt{B} \geq 5$. We find that a five sigma deviation is expected for WIMP masses,

$$
\begin{aligned}
\text { Tevatron : } & M_{X} \lesssim 15 \mathrm{GeV} \\
14 \mathrm{TeV} \text { LHC : } & M_{X} \lesssim 275 \mathrm{GeV} .
\end{aligned}
$$

If no deviation is observed, 95\% C.L. limits may be obtained,

$$
\begin{aligned}
\text { Tevatron }: & M_{X} \gtrsim 25 \mathrm{GeV} \\
14 \mathrm{TeV} \text { LHC }: & M_{X} \gtrsim 450 \mathrm{GeV} .^{3}
\end{aligned}
$$

One can also relax the assumption that $X$ is a thermal relic and determine limits in the $M_{X}-G_{A}$ parameter plane.

\section{CONCLUSIONS}

In this paper we have explored the idea that the dark matter is a cold thermal relic, and it is the only beyond the standard-model particle relevant for dark matter that is within the reach of the LHC, i.e., a maverick particle. We have employed a particular well motivated effective field theory description of the WIMP interactions with standard-model particles - Eq. (2) - which specifies the cosmological/astrophysical annihilation, scattering with nuclei, and collider

\footnotetext{
3 The corresponding WIMP mass limits for an LHC run with $\sqrt{s}=7 \mathrm{TeV}$ and a total integrated luminosity of $\int L \mathrm{dt}=1 \mathrm{fb}^{-1}$ are $M_{X}=40 \mathrm{GeV}$ for a five-sigma detection, and $M_{X}=55 \mathrm{GeV}$ for a $95 \%$ c.l. limit.
} 

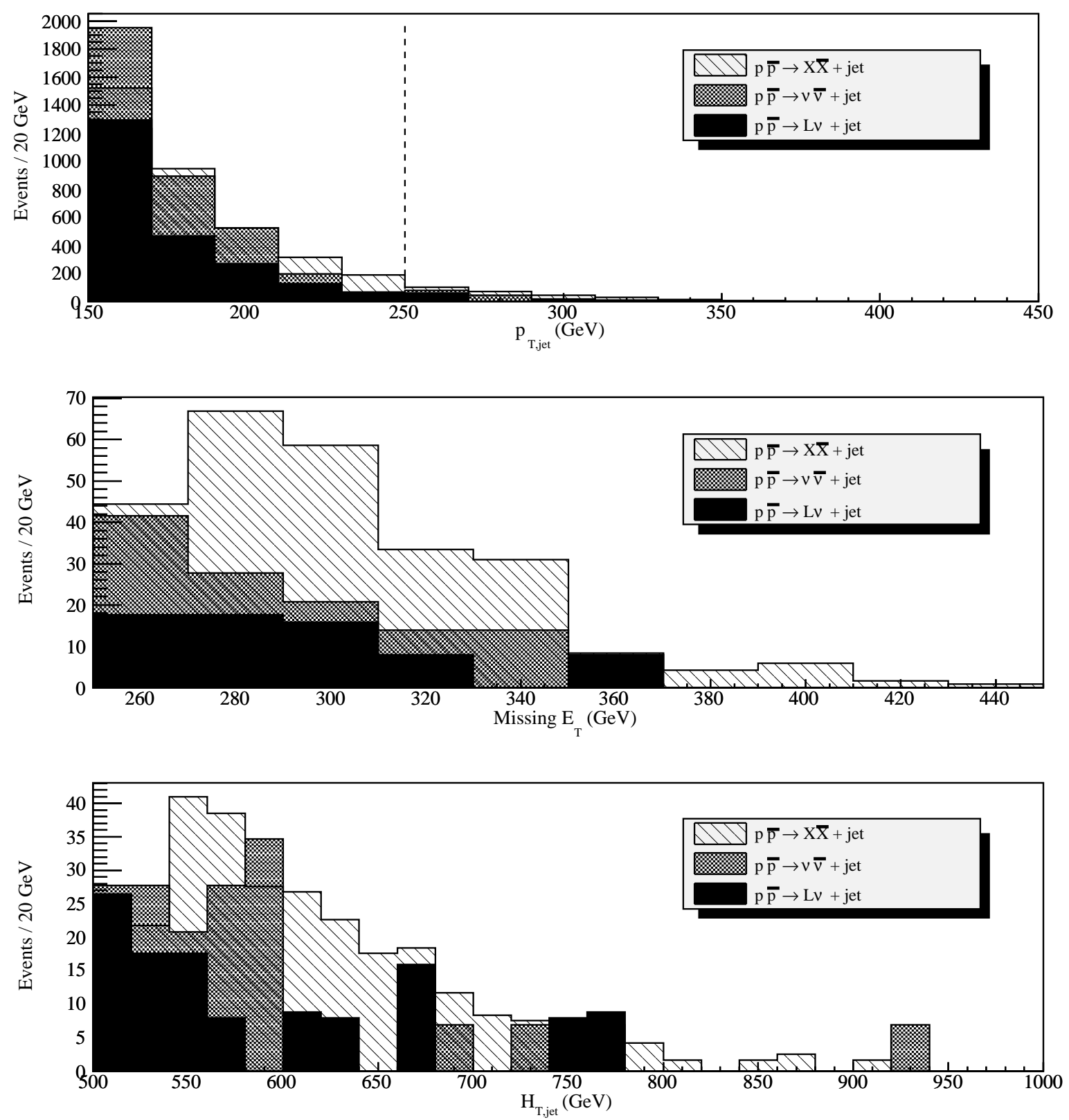

FIG. 6: The number of events as a function of $p_{T \text {,jet }}$ (top), $H_{T}$ (middle), and $\mathbb{E}_{T}$ (bottom) for WIMP production and standardmodel background processes at the Tevatron with $\sqrt{s}=1.96 \mathrm{TeV}$ with $\int L \mathrm{dt}=10 \mathrm{fb}^{-1}$, and a WIMP mass $M_{X}=5 \mathrm{GeV}$. The dotted line in the upper panel indicates the cut we impose on $p_{T \text {,jet }}$.

production of the WIMP. While the formalism is general, we explore the case of a Dirac WIMP interacting through an axial-vector interaction, which leads to spin-dependent low-energy WIMP-nucleon scattering, and consistent with the up until now null results of direct-detection experiments [8]. However, it is worth bearing in mind that for WIMPs with masses greater than about $200 \mathrm{GeV}$, vector and scalar interactions are also compatible with direct detection limits. Since the corresponding values of $G$ motivated by the relic density for those cases are smaller, the signals for a thermal relic will be somewhat more challenging to pick out from the backgrounds. It would be interesting to pursue those cases as well in future work.

We have further chosen flavor-independent coupling to quarks, as might be expected in a theory which does not lead to unacceptably large corrections to SM flavor observables. In fact, provided the WIMP has significant coupling to the up and down quarks which are present as valence quarks in the proton, we do not expect relaxing that assumption to change our results very significantly. We have also assumed no direct coupling to leptons. If there were coupling to 

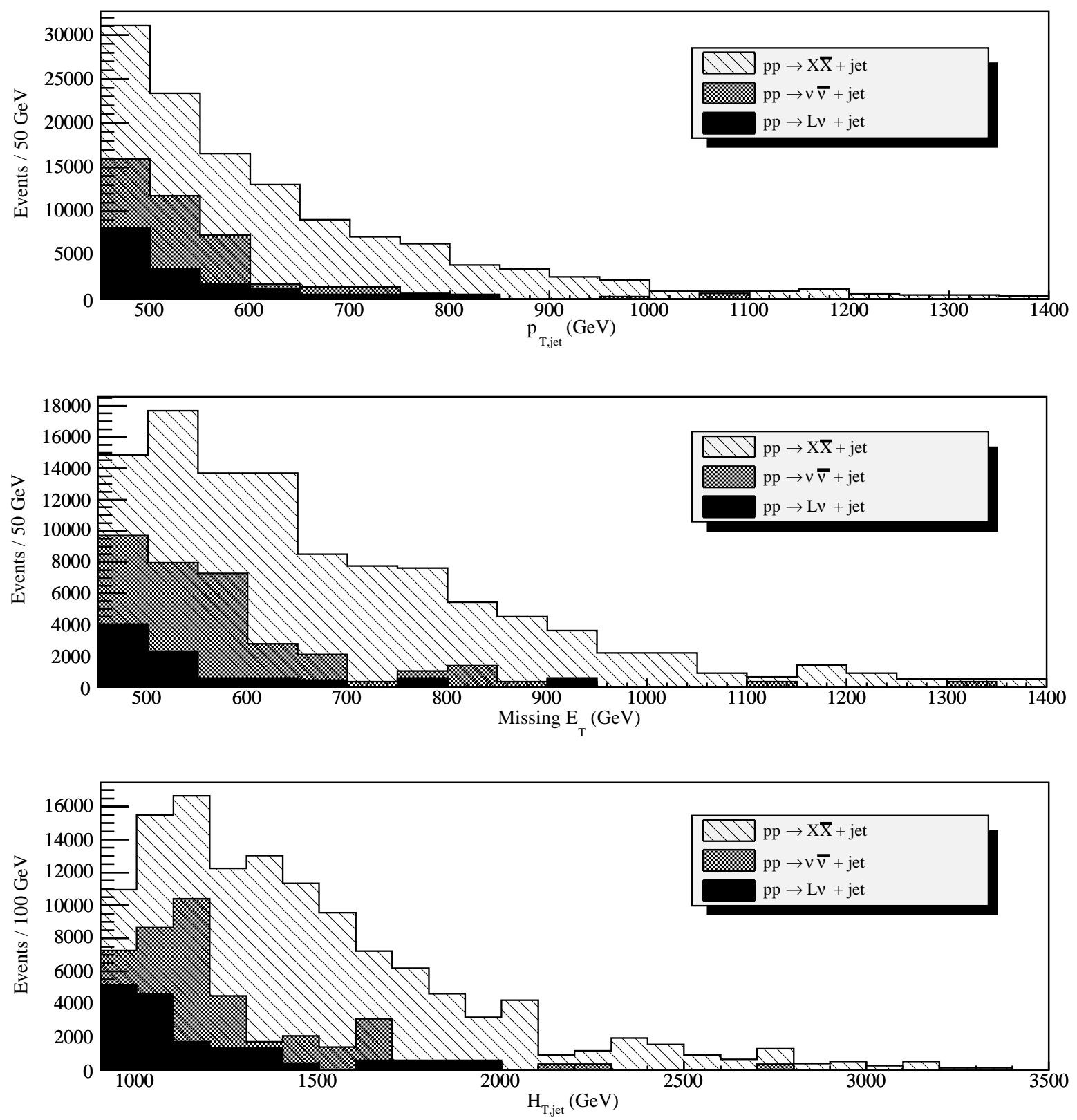

FIG. 7: The number of events as a function of $p_{T \text {,jet }}$ (top), $H_{T}$ (middle), and $\mathbb{E}_{T}$ (bottom) for WIMP production and the most important standard-model background processes at the LHC with $\sqrt{s}=14 \mathrm{TeV}$ with $\int L \mathrm{dt}=100 \mathrm{fb}^{-1}$, and a WIMP mass $M_{X}=50 \mathrm{GeV}$.

leptons, then depending on the UV completion, there may also be new contributions to processes such as $q \bar{q} \rightarrow \ell^{+} \ell^{-}$. Such a process has excellent discovery prospects at the LHC, and would provide information about the greater context in which the WIMP lives, but is model-dependent and thus not part of a minimal signal of maverick dark matter.

Here, we explore the possibility of production and detection at colliders of a pair of maverick WIMPs together with hard jets, $p p(p \bar{p}) \rightarrow X \bar{X}+$ jets. We find that the associated jets tend to have larger transverse momentum and missing transverse energy compared to background events, providing potential handles that can be exploited to extract the signal from the background. In particular, the numbers of jets, as well as differences in the number of events as a function of leading jet transverse momenta, $p_{T, \text { jet }}$, the scalar sums of jet transverse momenta plus missing transverse energy, $H_{T}=\sum\left|\vec{p}_{T}\right|+\mathbb{E}_{T}$, and the missing transverse energy, $\mathbb{E}_{T}$, differs between signal and background. This leads to a promising discovery potential for this class of maverick WIMPs. Because our WIMPs are produced through higher dimensional operators without the appearance of intermediate on-shell colored particles, we are lead 
to devise somewhat different analysis strategies than those typically employed for e.g., searches for supersymmetry or large extra dimensions.

We estimate the discovery mass reach of the Tevatron with $10 \mathrm{fb}^{-1}$ total integrated luminosity to be about 15 $\mathrm{GeV}$ and the mass reach of the $14 \mathrm{TeV}$ LHC with an integrated luminosity of $100 \mathrm{fb}^{-1}$ (roughly a year's running at design luminosity) to be $275 \mathrm{GeV}$. These numbers are rough estimates, for there are three issues we did not properly account for: 1) We have not seriously attempted to optimize the cuts, which would require a more detailed detector simulation, and more sophisticated simulation of the backgrounds (ideally using data as input); 2) Using $S / \sqrt{B}$ as an indication of the mass reach is reasonable for a counting experiment, but ideally one would perform a shape analysis, making use of the fact that the background and signal lead to different $p_{T}$ and $H_{T}$ distributions; and 3) We have ignored uncertainties in the background calculation.

Properly taking into account issues 1) and 2) above would result in a better limit, while issue 3) would weaken the limit. To get some idea of the uncertainty related to issue 3), we can compare to the CDF analysis in Ref. 20] based on $368 \mathrm{pb}^{-1}$ of $p \bar{p}$ collisions. With the cuts employed in their analysis, they expect a SM background of 265 \pm 30 events (including $15 \pm 10$ QCD multijet events), and observe 263 events. Using the Bayesian approach of Ref. 23] results in a maximum number of 67 signal events from new physics (including a $13 \%$ uncertainty in the signal acceptance). Using the same cuts as in the CDF analysis, we would expect 67 maverick dark matter events for a mass of $14 \mathrm{GeV}$. Our naïve $S / \sqrt{B}$ analysis would result in a mass limit of $11 \mathrm{GeV}$. This leads us to believe that our projected limits may not be too far off what is achievable (and in fact may be quite conservative). It also suggests that the QCD multijet background may not be an issue.

Ultimately, we expect that it will be difficult to push the mass reach beyond $15 \mathrm{GeV}$ and $400 \mathrm{GeV}$, for the Tevatron and LHC respectively, because there is simply not enough energy available to create pairs of more massive WIMPs.

Missing transverse energy searches have been proposed as a strategy to explore several scenarios for BSM physics. A question for our analysis (in fact, a question for all WIMP searches at colliders) is that if a missing transverse energy signal that cannot be described by standard-model physics is detected, how do we know it is related to dark matter? One possible answer comes in the form of a consistency check: the production cross section depends upon both the mass of the WIMP, $M_{X}$, and its coupling to quarks, $G_{A}$. If the maverick WIMP is the dark matter, we can infer $G_{A}\left(M_{X}\right)$ from the relic density calculation. The kinematics of the event distribution (such as the peak in the $p_{T, \text { jet }}$ distribution) carries information about $M_{X}$. One can ask whether the combination of $G_{A}\left(M_{X}\right)$ for dark matter and $M_{X}$ from kinematical determinations agrees with the measured total production cross section. ${ }^{4}$ In addition, if signals of direct or indirect detection become evident, one can also correlate with them.

A maverick scenario of dark matter is interesting, and we have demonstrated that collider experiments can constrain (or discover!) maverick dark matter.

\section{Acknowledgments}

We would like to thank Florencia Canelli, John Conway, Jonathan Feng, Henry Frisch, JoAnne Hewett, Ben Kiliminster, Tom LeCompte, Frank Petriello, Tilman Plehn, Will Shepherd, Jay Wacker, and Lian-tao Wang for useful discussions. This work was supported in part by the Department of Energy at the University of Chicago and Fermilab. T. Tait is grateful to the SLAC theory group for their hospitality during his many visits.

[1] E. Komatsu et al., arXiv:1001.4538 [astro-ph.CO].

[2] H. Goldberg, Phys. Rev. Lett. 50, 1419 (1983); J. R. Ellis, J. S. Hagelin, D. V. Nanopoulos, K. A. Olive and M. Srednicki, Nucl. Phys. B 238, 453 (1984); G. L. Kane, C. F. Kolda, L. Roszkowski and J. D. Wells, Phys. Rev. D 49, 6173 (1994) arXiv:hep-ph/9312272.

[3] K. Agashe and G. Servant, Phys. Rev. Lett. 93, 231805 (2004) arXiv:hep-ph/0403143]; JCAP 0502, 002 (2005) arXiv:hep-ph/0411254]; K. Agashe, A. Falkowski, I. Low and G. Servant, arXiv:0712.2455 [hep-ph].

[4] E. W. Kolb and R. Slansky, Phys. Lett. B 135, 378 (1984); G. Servant and T. M. P. Tait, Nucl. Phys. B 650, 391 (2003) arXiv:hep-ph/0206071] H. C. Cheng, J. L. Feng and K. T. Matchev, Phys. Rev. Lett. 89, 211301 (2002) arXiv:hep-ph/0207125; D. Hooper and S. Profumo, Phys. Rept. 453, 29 (2007) arXiv:hep-ph/0701197.

[5] G. Bertone, D. Hooper, and J. Silk, Phys. Rep. 405, 279 (2005).

\footnotetext{
${ }^{4}$ If the two match, it will be highly suggestive of a discovery of dark matter. If they do not, one will be left wondering if the new signal of missing energy is unrelated to dark matter, or if the WIMP is perhaps not a thermal relic.
} 
[6] H. Baer, C. Balasz, A. Belyaev, T. Krupovnickas, and X. Tata, JHEP 0306, 054 (2003).

[7] A. Abulencia et al., Phys. Rev. Lett. 97, 171802 (2006).

[8] M. Beltran, D. Hooper, E. W. Kolb, and Z. A. C. Krusberg, Phys. Rev. D 80, 043509 (2009).

[9] A. Birkedal, K. Matchev and M. Perelstein, Phys. Rev. D 70, 077701 (2004) arXiv:hep-ph/0403004; P. Konar, K. Kong, K. T. Matchev and M. Perelstein, New J. Phys. 11, 105004 (2009) arXiv:0902.2000 [hep-ph]].

[10] J. L. Feng, S. Su and F. Takayama, Phys. Rev. Lett. 96, 151802 (2006) arXiv:hep-ph/0503117].

[11] Q. H. Cao, C. R. Chen, C. S. Li and H. Zhang, arXiv:0912.4511 [hep-ph].

[12] W. Shepherd, T. M. P. Tait and G. Zaharijas, Phys. Rev. D 79, 055022 (2009) arXiv:0901.2125 [hep-ph]].

[13] E. W. Kolb and M. S. Turner, The Early Universe (Westview Press, Boulder, CO, 1994).

[14] M. Herquet and F. Maltoni, Nucl. Phys. B (Proc. Suppl.) 179-180, 211 (2008).

[15] T. Sjostrand, S. Mrenna, and P. Skands, JHEP 0605, 026 (2006).

[16] http://www.physics.ucdavis.edu/ conway/research/software/pgs/pgs4-general.htm

[17] J. M. Campbell, L. W. Huston, and W. J. Stirling, Rep. Prog. Phys. 70, 89 (2007).

[18] W. Beenakker, W. L. van Neerven, R. Meng, G. A. Schuler and J. Smith, collisions," Nucl. Phys. B 351, 507 (1991).

[19] G. L. Bayatian et al. [CMS Collaboration], J. Phys. G 34, 995 (2007).

[20] A. Abulencia et al. [CDF Collaboration], Phys. Rev. Lett. 97, 171802 (2006) arXiv:hep-ex/0605101].

[21] S. Catani, F. Krauss, R. Kuhn and B. R. Webber, JHEP 0111, 063 (2001) arXiv:hep-ph/0109231; S. Hoche, F. Krauss, N. Lavesson, L. Lonnblad, M. Mangano, A. Schalicke and S. Schumann, arXiv:hep-ph/0602031.

[22] J. Alwall, M. P. Le, M. Lisanti and J. G. Wacker, Phys. Lett. B 666, 34 (2008) [arXiv:0803.0019 [hep-ph]]; J. Alwall, M. P. Le, M. Lisanti and J. G. Wacker, Phys. Rev. D 79, 015005 (2009) arXiv:0809.3264 [hep-ph]].

[23] J. Heinrich, C. Blocker, J. Conway, L. Demortier, L. Lyons, G. Punzi, and P. K. Sinervo, arXiv:physics/0409129. 